\title{
Foundation Medicine Inc
}

National Cancer Institute

\section{Source}

National Cancer Institute. Foundation Medicine Inc. NCI Thesaurus. Code C133726.

A biotechnology company which develops and manufactures genomic analysis

diagnostics based on next-generation sequencing technology for detection of solid and circulating cancers. 